\title{
Nonlinear condensation modes
}

\author{
J A N W E I L A N D \\ Chalmers University of Technology and EURATOM-VR Association, \\ S-41296 Gothenburg, Sweden \\ (elfjw@chalmers.se)
}

(Received 30 November 2009 and accepted 13 December 2009, first published online 26 January 2010)

\begin{abstract}
In the far edge, in L-mode plasmas and the outer parts of the H-mode barrier, tokamak plasmas may be so collisional that parallel electron motion can be neglected or included as a perturbation. Thus we have a regime that is basically two dimensional. We will here consider the outer part of the $\mathrm{H}$-mode barrier where transport is small. Recently a condensation mode was found for this regime. We have extended the theory to the nonlinear regime.
\end{abstract}

\section{Introduction}

The interest in the theory of the edge region of tokamaks was greatly increased after the discovery of the H-mode in 1982 [1]. Reactive linear eigenmodes of the type that we study here were actually discovered in 1991 for steep gradients in a system of ion temperature gradient (ITG) modes and trapped electron (T-E) modes [2]. Since the H-mode barrier usually starts just inside the separatrix, its outer parts should be in a strongly collisional regime. Thus interest is focused on possible instabilities in this regime, including the reactive electrostatic ballooning mode found by Novakovskii et al. in 1995 [3]. This mode becomes a reactive twodimensional mode when the collisionality is so strong that it completely prevents electrons from moving along the magnetic field lines or for large mode numbers. This is a strongly unstable mode with a growth rate of the order of the ideal MHD magnitude. This mode is actually recovered in the same mathematical form as for trapped fraction equal to 1 in the system of toroidal ITG modes and T-E modes mentioned above. It can be stabilized by finite Larmor radius (FLR) effects [2] and was found to be important in turbulence simulations by Rogers et al. $[4,5]$. The results also compared favourably with experiment [6].

In a later work most of the results of Rogers et al. were also recovered analytically [7], and it was also found that the reactive mode, discussed above, also persists for lower collisionality if the mode number is high.

The L-H transition due to the FLR stabilization of the Novakovskii mode was studied in detail in [8], including comparison with ITG modes. In the FLR-stabilized regime (H-mode), a mode with a growth rate of the order of the magnetic drift frequency was found both for the ITG-TE system [2] and the collision-dominated case [9]. This mode gives a small transport compatible with $\mathrm{H}$-mode and also gives a particle pinch. Both the ITG-TE system and the collision-dominated system were reviewed in [10], including a comparison of $\mathrm{H}$-mode thresholds due to stabilization by FLR and shear flows. Later a generalized formalism with a complex trapped fraction was shown to be able to make the transition from the drift wave system 
to the collision-dominated system [11]. In this transition, the typical separation in two modes remained for the whole transition. Thus it could apply to the whole $\mathrm{H}$-mode barrier.

\section{Basic equations}

For ions we use the following energy equation:

$$
\frac{3}{2} n\left(\frac{\partial}{\partial t}+\mathbf{v}_{\mathbf{i}} \cdot \nabla\right) T_{\mathrm{i}}+P_{\mathrm{i}} \nabla \cdot \mathbf{v}_{\mathbf{i}}=-\nabla \cdot \mathbf{q}_{\mathbf{i}}
$$

with

This leads to

$$
\mathbf{q}_{\mathrm{i}}=\mathbf{q}_{* \mathrm{i}}=\frac{5}{2} \frac{P}{m_{\mathrm{i}} \Omega_{\mathrm{ci}}}\left(\widehat{\mathbf{e}}_{\|} \times \nabla T_{\mathrm{i}}\right)
$$

$$
\frac{\delta T_{\mathrm{i}}}{T_{\mathrm{i}}}=\frac{\omega}{\omega-\frac{5}{3} \omega_{D_{\mathrm{i}}}}\left[\frac{2}{3} \frac{\delta n_{\mathrm{i}}}{n}+\frac{\omega_{* \mathrm{e}}}{\omega}\left(\eta_{\mathrm{i}}-\frac{2}{3}\right) \frac{e \phi}{T_{\mathrm{e}}}\right] .
$$

In a strongly collisional, Braghinskii regime, we may write the parallel electron current as

$$
\begin{gathered}
j_{\| \mathrm{e}\rangle}=e n D_{\mathrm{e}} \widehat{\mathbf{e}}_{\|} \cdot\left(\frac{1}{n} \nabla_{\|} n_{\mathrm{e}}-\frac{e}{T_{\mathrm{e}}} \nabla_{\|} \phi+1.71 \frac{\nabla_{\|} T_{\mathrm{e}}}{T_{\mathrm{e}}}\right) \\
D_{\mathrm{e}}=\frac{2 T_{\mathrm{e}}}{m_{\mathrm{e}} \nu_{\mathrm{ei}}} .
\end{gathered}
$$

Then using the same reactive parts of the electron energy equation as for ions, we arrive at

$$
\frac{\delta T_{\mathrm{e}}}{T_{\mathrm{e}}}=\frac{\omega}{\omega-\frac{5}{3} \omega_{D_{\mathrm{e}}}+i \nu_{\mathrm{T}}}\left[\frac{2}{3} \frac{\delta n_{\mathrm{e}}}{n}+\frac{\omega_{* \mathrm{e}}}{\omega}\left(\eta_{\mathrm{e}}-\frac{2}{3}\right) \frac{e \phi}{T_{\mathrm{e}}}\right],
$$

where

$$
\nu_{\mathrm{T}}=1.06 k_{\|}^{2} D_{\mathrm{e}} .
$$

The density perturbations take the forms

$$
\begin{gathered}
\frac{\delta n_{\mathrm{i}}}{n}=\frac{\omega\left(\omega_{\bullet \mathrm{e}}-\omega_{D_{\mathrm{e}}}\right)+\left(\eta_{\mathrm{i}}-\frac{7}{3}+\frac{5}{3} \varepsilon_{\mathrm{n}}\right) \omega_{* \mathrm{e}} \omega_{D_{\mathrm{e}}}+\mathrm{FL}}{\omega^{2}-\frac{10}{3} \omega \omega_{D_{\mathrm{i}}}+\frac{5}{3} \omega_{D_{\mathrm{i}}}^{2}} \frac{e \phi}{T_{\mathrm{e}}} \\
\mathrm{FL}=-k^{2} \rho^{2}\left(\omega-\omega_{* \mathrm{i}}\right)\left(\omega-\frac{5}{3} \omega_{D_{\mathrm{i}}}\right) \\
\frac{\delta n_{\mathrm{e}}}{n}=\frac{\omega\left(\omega_{\bullet \mathrm{e}}-\omega_{D_{\mathrm{e}}}\right)+\left(\eta_{\mathrm{e}}-\frac{7}{3}+\frac{5}{3} \varepsilon_{\mathrm{n}}\right) \omega_{* \mathrm{e}} \omega_{D_{\mathrm{e}}}+i k_{\|}^{2} D_{\mathrm{e}} T(\omega)}{\omega^{2}-\frac{10}{3} \omega \omega_{D_{\mathrm{e}}}+\frac{5}{3} \omega_{D_{\mathrm{e}}}^{2}+i k_{\|}^{2} D_{\mathrm{e}} N(\omega)} \frac{e \phi}{T_{\mathrm{e}}}
\end{gathered}
$$

where

$$
\begin{gathered}
T(\omega)=\omega-\frac{5}{3} \omega_{D_{\mathrm{e}}}-1.71 \omega_{*_{\mathrm{e}}}\left(\eta_{\mathrm{e}}-\frac{2}{3}\right)+1.06\left(\omega_{*_{\mathrm{e}}}-\omega_{D_{\mathrm{e}}}\right) \\
N(\omega)=\omega-\frac{5}{3} \omega_{D_{\mathrm{e}}}+1.14 \omega+1.06\left(\omega-\omega_{D_{\mathrm{e}}}\right) .
\end{gathered}
$$

Now quasineutrality leads to an eigenvalue equation that is quartic in $\omega$. This is actually the simplest way to derive an eigenvalue equation. We now consider edge parameters where $\varepsilon_{\mathrm{n}} \ll 1$. The derivation is further simplified by assuming 
$\eta_{\mathrm{i}} \sim \eta_{\mathrm{e}} \sim 1 / \varepsilon_{\mathrm{n}}$. However, this assumption can be relaxed. For the present derivation, we will make use of the large $\eta$ ordering. As it turns out, the quartic dispersion relation (taking the local limit) in this ordering leads to two quadratic dispersion relations: one with $\omega \sim \omega_{*}$ (high frequency) and one with $\omega \sim \omega_{D}$ (low frequency). These modes are both mixed between ITG and T-E modes. The high-frequency mode also has higher growth rate and dominates in L-mode. The low-frequency mode is, on the other hand, compatible with $\mathrm{H}$-mode. The transition in this system is caused by FLR stabilization. The low-frequency mode takes the form of a condensation mode $\delta P=0$ when FLR and ion inertia can be ignored. This can easily be seen from the condition of vanishing parallel current as

$$
\nabla \cdot \mathbf{j}_{\perp}=0 .
$$

Here the ExB drifts cancel (also the nonlinear effects) and the only remaining drifts are the ion polarization and diamagnetic drifts. The latter give magnetic drifts and pressure perturbation. We now include the Hasegawa-Mima nonlinearity by modifying FL in (5b) by adding

$$
\mathrm{FL} \rightarrow \mathrm{FL}+\left(\omega-\frac{5}{3} \omega_{D_{\mathrm{i}}}\right) \frac{1}{\Omega_{c}} \mathbf{v}_{\mathbf{E}} \cdot \nabla \Delta \phi .
$$

The dielectric function will have the following form:

$$
\varepsilon(\omega, k)=\frac{1}{k^{2} \lambda_{D_{\mathrm{e}}}^{2}} \frac{\left(\frac{\delta n_{\mathrm{i}}}{n}-\frac{\delta n_{\mathrm{e}}}{n}\right)}{\frac{e \phi}{T_{\mathrm{e}}}} .
$$

The nonlinear equation can in general be written as

$$
\omega \varepsilon(\omega, k) E_{\omega, k}=-\frac{i}{\varepsilon_{0}} j_{\omega, k}^{(2)},
$$

where

$$
\nabla \cdot \mathbf{j}^{(2)}=e\left[n \nabla \cdot \mathbf{v}_{\mathrm{pi}}+\frac{1}{T_{\mathrm{i}}} \mathbf{v}_{\mathrm{D}_{\mathrm{i}}} \cdot \nabla \delta p_{\mathrm{i}}^{(2)}-\frac{1}{T_{\mathrm{e}}} \mathbf{v}_{\mathrm{D}_{\mathrm{e}}} \cdot \nabla \delta p_{\mathrm{e}}^{(2)}\right] .
$$

We now need the divergence of the current in order to eliminate the Laplacian that will be generated by the denominator in (8). Thus, in order to get more tractable expressions, we eliminate the denominators coming from (5a) and (6a), ignoring parallel electron conductivity to get the following nonlinear equation:

$$
D(\omega, k) \phi_{\omega, k}=i \Gamma(\omega, k) \frac{1}{T_{\mathrm{i}}} \mathbf{v}_{\mathrm{D}_{\mathrm{i}}} \cdot \nabla\left(\delta p_{\mathrm{i}}^{(2)}+\delta p_{\mathrm{e}}^{(2)}\right)
$$

where

$$
\begin{aligned}
D(\omega)= & \frac{5}{3} \omega \omega_{*_{\mathrm{e}}} \omega_{D_{\mathrm{e}}}\left(1+\frac{1}{\tau}\right)\left(2 \omega-\omega_{D_{\mathrm{e}}}\left(1-\frac{1}{\tau}\right)\right)+\omega^{2} \omega_{*_{\mathrm{e}}}\left(\eta_{\mathrm{e}} \omega_{D_{\mathrm{e}}}-\eta_{\mathrm{i}} \omega_{D_{\mathrm{i}}}\right) \\
& +\frac{10}{3 \tau} \omega \omega_{*_{\mathrm{e}}} \omega_{D_{\mathrm{e}}}^{2}\left(\eta_{\mathrm{e}}-\eta_{\mathrm{i}}\right)-\frac{5}{3 \tau} \omega_{*_{\mathrm{e}}} \omega_{D_{\mathrm{e}}}^{2}\left(\eta_{\mathrm{e}} \omega_{D_{\mathrm{i}}}-\eta_{\mathrm{i}} \omega_{D_{\mathrm{e}}}\right)
\end{aligned}
$$

and

$$
\Gamma(\omega)=\left(\omega^{2}-\frac{10}{3} \omega \omega_{D_{\mathrm{i}}}+\frac{5}{3} \omega_{D_{\mathrm{i}}}^{2}\right)\left(\omega^{2}-\frac{10}{3} \omega \omega_{D_{\mathrm{e}}}+\frac{5}{3} \omega_{D_{\mathrm{e}}}^{2}\right) .
$$

Because we have assumed strong temperature gradients, we now approximate

$$
\delta p^{(2)} \approx \delta n \delta T \text {. }
$$


We note that the form of the electron temperature perturbation turns into that of ions in the limit of large collisionality. The nonlinear equation obtained here can also be obtained from the general set of nonlinear equations in collision-dominated plasmas derived by Shukla and Weiland [12].

\section{Linear properties}

The linear properties of our low-frequency mode are determined through the linear dispersion relation:

$$
D(\omega)=0 .
$$

The resulting dispersion relation can be written as

$$
\omega^{2}-\frac{10}{3} \xi \omega_{D_{\mathrm{e}}} \omega+\frac{5}{3} \delta \omega_{D_{\mathrm{e}}}^{2}=i \gamma_{\mathrm{D}}^{2},
$$

where $\gamma_{\mathrm{D}}$ is a damping due to parallel motion and will here be assumed to be small. The coefficients are

$$
\xi=\frac{\eta_{\mathrm{i}}-\eta_{\mathrm{e}}+\tau / 2-1 /(2 \tau)}{\eta_{\mathrm{i}}+\tau \eta_{\mathrm{e}}+1+\tau} \quad \text { and } \quad \delta=\frac{\eta_{\mathrm{i}}+(1 / \tau) \eta_{\mathrm{e}}-7(1+1 / \tau) / 3}{\eta_{\mathrm{i}}+\tau \eta_{\mathrm{e}}+1+\tau} .
$$

The local part of (13) has the solution:

$$
\omega=\frac{5}{3} \xi \omega_{D_{\mathrm{e}}} \pm \frac{5}{3} \omega_{D_{\mathrm{e}}} \sqrt{\xi^{2}-\frac{3}{5} \delta}
$$

Marginal stability occurs at

$$
\eta_{\mathrm{i}}+\frac{1}{\tau} \eta_{\mathrm{e}}>\frac{7}{3}\left(1+\frac{1}{\tau}\right)
$$

We note that the direction of propagation depends on the relation between electron and ion temperature gradients with zero frequency for equal temperatures and gradients.

\section{Nonlinear properties}

We now return to (9a). Considering the case of weak nonlinearity, we can expand $D(\omega)$ around the linear eigenfrequency, i.e.

$$
\omega \rightarrow \omega_{\mathrm{L}}+i \frac{\partial}{\partial \hat{t}},
$$

where $\hat{t}$ denotes a slow time variation and

$$
D(\omega) \approx D\left(\omega_{\mathrm{L}}\right)+\frac{\partial D}{\partial \omega} i \frac{\partial}{\partial \hat{t}},
$$

with

$$
\frac{\partial D}{\partial \omega}=\omega \omega_{D_{\mathrm{e}}}^{2}\left\{\varpi\left[\frac{20}{3}\left(1+\frac{1}{\tau}\right)+2\left(\eta_{\mathrm{e}}+\frac{1}{\tau} \eta_{i}\right)\right]-\frac{5}{3}\left(1-\frac{1}{\tau^{2}}\right)+\frac{10}{3 \tau}\left(\eta_{\mathrm{e}}-\eta_{\mathrm{i}}\right)\right\}
$$

and

$$
\varpi=\frac{\omega}{\omega_{D_{\mathrm{e}}}}
$$


Now this coefficient will vanish for:

$$
\varpi_{\mathrm{r}}=\frac{\frac{10}{3 \tau}\left(\eta_{\mathrm{e}}-\eta_{\mathrm{i}}\right)-\frac{5}{3}\left(1-\frac{1}{\tau^{2}}\right)}{\frac{20}{3}\left(1+\frac{1}{\tau}\right)+2\left(\eta_{\mathrm{e}}+\frac{1}{\tau} \eta_{\mathrm{i}}\right)} .
$$

Our nonlinear equation is now written as

$$
\frac{\partial \hat{\phi}}{\partial \hat{t}}=\frac{\Gamma(\omega)}{\partial D / \partial \omega} \frac{e}{T_{\mathrm{i}}} \mathbf{v}_{\mathrm{D}_{\mathrm{i}}} \cdot \nabla\left(\delta n_{\mathrm{i}} \delta T_{\mathrm{i}}+\delta n_{\mathrm{e}} \delta T_{\mathrm{e}}\right) .
$$

In the present limit of large temperature gradients, we have

$$
\frac{\delta T_{\mathrm{j}}}{T_{\mathrm{j}}}=\frac{\eta_{\mathrm{j}} \omega_{* \mathrm{e}}}{\omega-\frac{5}{3} \omega_{D_{\mathrm{j}}}} \frac{e \phi}{T_{\mathrm{e}}}
$$

and

$$
\frac{\delta n_{\mathrm{j}}}{n_{\mathrm{j}}}=\frac{\eta_{\mathrm{j}} \omega_{*_{\mathrm{e}}} \omega_{D_{\mathrm{j}}}}{\omega^{2}-\frac{10}{3} \omega \omega_{D_{\mathrm{j}}}+\frac{5}{3} \omega_{D_{\mathrm{j}}}^{2}} \frac{e \phi}{T_{\mathrm{e}}} .
$$

These expressions should be substituted into (21) in order to obtain a well-defined nonlinear equation. Because of the form of $\Gamma(\omega)$, the denominators of the density perturbations will be cancelled thus simplifying the nonlinear equation. Here $D(\omega)$ represents the linear dispersion function and its derivative with respect to frequency gives the wave energy. It is interesting to note that the linear growth rate has its maximum for purely growing modes, and we have this when the temperature gradients of electrons and ions are equal for equal temperatures. This case also corresponds to zero wave energy at marginal stability according to (17). We would in this case have to continue the expansion to second-order derivative. This is a typical property seen in most systems. We have here strictly applied our ordering to the nonlinear terms. We note, however, that the Hasegava-Mima nonlinearity $[13]$ enters to next order. Thus, in practice there will be a dual cascade. Since $\Gamma(\omega)$ does not depend on the temperature gradients, we see that a change of direction of propagation near $D(\omega)=0$ would not change the sign of the coupling factor. Thus we could change the total sign of the nonlinear couplings so that we can get nonlinear instability [14].

\section{Discussion}

In this paper, we considered the nonlinear evolution of a type of condensation mode previously found to occur on the H-mode barrier after FLR stabilization. The condensation property is obtained only in the linear local limit and is expressed as

$$
\delta P=0 .
$$

However, the mode retains its condensation nature when the non-local and nonlinear effects are small and we can then study its evolution under more realistic conditions. We found possibility for both nonlinear instability and dual cascade. This could potentially influence the stability of an H-mode. An interesting property of the mode, found already in previous linear studies, is that the diamagnetic heat flow is needed for instability. Thus, quadratic curvature effects are needed, although $\varepsilon_{n} \ll 1$. The reason for this is that the mode frequency is of the order of the magnetic 
drift frequency, although the diamagnetic drift frequency is much higher. This property is due to the separation of the general quartic dispersion relation into two quadratic dispersion relations for an edge ordering. Another special property is that the dominant nonlinearity is due to magnetic inhomogeneity.

\section{References}

[1] Wagner, F. et al. 1982 Phys. Rev. Lett. 53, 1453.

[2] Weiland, J. and Nordman, H. 1991 Nucl. Fusion 31, 390.

[3] Novakovskii, S. V., Guzdar, P. N., Drake, J. F., Liu, C. S. and Waelbroeck, F. 1995 Phys. Plasmas 2, 2381.

[4] Rogers. B. N., Drake, J. F. and Zeiler, A. 1998 Phys. Rev. Lett. 81, 4396.

[5] Rogers, B. N. and Drake, J. F. 1999 Phys. Plasmas 6, 2797.

[6] Hubbard, A. E., Boivin, R. L., Drake, J. F., Greenwald, M., In, Y., Irby, J. H., Rogers, B. N. and Snipes, J. A. 1998 Plasma Phys. Control. Fusion 40, 689.

[7] Singh, R., Tangri, V., Kaw, P. and Guzdar, P. N. 2005 Phys. Plasmas 12, 092307.

[8] Singh, R., Nordman, H., Anderson, J. and Weiland, J. 1998 Phys. Plasmas 5, 3669.

[9] Singh, R. and Weiland, J. 1999 Phys. Plasmas 6, 1397.

[10] Weiland, J. 2000 Collective Modes in Inhomogeneous Plasmas, Kinetic and Advanced Fluid theory. Bristol: IOP Publishing.

[11] Moestam, R. and Weiland, J. 2002 Nucl. Fusion 42, 663.

[12] Shukla, P. K. and Weiland, J. 1989 Phys. Rev. A40, 341.

[13] Hasegawa, A. and Mima, K. 1978 Phys Fluids 21, 87.

[14] Weiland, J. and Wilhelmsson, H. 1977 Coherent Non-Linear Interaction of Waves in Plasmas. Oxford: Pergamon Press. 